Pacific Journal of Mathematics

COMPLETELY SEMISIMPLE INVERSE $\triangle$-SEMIGROUPS 


\title{
COMPLETELY SEMISIMPLE INVERSE $\triangle$-SEMIGROUPS ADMITTING PRINCIPAL SERIES
}

\author{
P. G. Trotter and TAKayuki Tamura
}

\begin{abstract}
A $\Delta$-semigroup is a semigroup whose lattice of congruences is a chain with respect to inclusion. A completely semisimple inverse $\Delta$-semigroup that admits a principal series is characterized here as a semigroup that results from a particular series of ideal extensions of Brandt semigroups by Brandt semigroups. A characterization is given of finite inverse $\Delta$-semigroups in terms of groups, Brandt semigroups, and one to one partial transformations of sets.
\end{abstract}

1. Introduction. A $\Delta$-semigroup is a semigroup whose lattice of congruences is a chain with respect to inclusion. Schein [8] and Tamura [11] showed that a commutative $\Delta$-semigroup is either a quasicyclic group $A$, or a commutative nil semigroup $B$ with the divisibility chain condition, or $A^{0}$, or $B^{1}$. We study here the structure of completely semisimple inverse $\Delta$-semigroups with principal series. Such semigroups will be characterized in terms of $\Delta$-groups, idempotent properties, and ideal extensions of Brandt semigroups by Brandt semigroups.

In [11] it was shown that the least semilattice congruence on a $\Delta$-semigroup has at most two classes. We begin by characterizing completely semisimple inverse semigroups admitting principal series and having this property.

In the final section we show that each finite inverse $\Delta$-semigroup determines a set of structure data that involves groups, Brandt semigroups and one to one partial transformations of sets. Conversely the semigroup can be reconstructed from the structure data.

2. Preliminaries. We call a semigroup $S$ an $\mathscr{S}_{1^{-}}$, or $\mathscr{S}_{2^{-}}$ semigroup if the smallest semilattice congruence on $S$ has one, or two congruence classes respectively. $S$ is a $\Delta$-semigroup only if it is an $\mathscr{Y}_{1}$ - or $\mathscr{I}_{2}$-semigroup. In this section we characterize completely semisimple inverse $\mathscr{S}_{1^{-}}$, or $\mathscr{S}_{2^{-}}$-semigroups that admit principal series.

A subsemigroup $H$ of a semigroup $S$ is $\mathscr{S}$-unitary if and only if whenever $H x y H \subseteq H$ for $x, y \in S^{1}$ then $H x, y H \subseteq H$. Notice that if $E$ is a semilattice and $e f g=e$ in $E$ then $e f=e=g e$. Hence, any class of a semilattice congruence on $S$ is $\mathscr{S}$-unitary. Let $\mathscr{J}^{*}$ denote the least congruence on $S$ containing Green's relation $\mathscr{J}$. For $a \in S$ let $J_{a}$ be the $\mathscr{J}$-class of $a$ and $J(a)=S^{1} a S^{1}$. 
THEOREM 2.1. Let $S$ be a regular semigroup. The following are equivalent :

(i) $S$ is an $\mathscr{S}_{1}$-semigroup.

(ii) $\mathscr{J}^{*}=S \times S$.

(iii) Each $\mathscr{S}$-unitary subsemigroup of $S$ that is a union of $\mathscr{J}$-classes is an ideal.

Proof. Howie and Lallement [2] have shown that $\mathscr{J}^{*}$ is the least semilattice congruence on $S$. Hence (i) and (ii) are equivalent.

(ii) implies (iii). Let $H$ be an $\mathscr{S}$-unitary subsemigroup that is a union of $\mathscr{J}$-classes but is not an ideal. Suppose $x a y \in H$ for some $x, y \in S^{1}, a \in S$. Then $H x a y H \subseteq H$ so $H x a \subseteq H$. Hence $H x a H \subseteq H$ so $a H \subseteq H$ and $H a H \subseteq H$. If $J_{a}=J_{b}, b \in S$, then there exists $r, s \in S^{1}$ so that $H x r b s y H \subseteq H$ and similarly $H b H \subseteq H$. So $H J_{a} H \subseteq H$. Since $H$ is not an ideal and is $\mathscr{S}$-unitary there is a $d \in S$ so that $H d H \not \subset H$. Define

$$
\begin{aligned}
& C_{h}=\left\{a \in S ; x J_{a} y \cap H \neq \square \text { for some } x, y \in S^{1}\right\} \text { and } \\
& \bar{C}_{H}=\left\{d \in S ; d \notin C_{H}\right\} .
\end{aligned}
$$

Let $\rho_{H}$ denote the equivalence relation on $S$ with classes $C_{H}$ and $\bar{C}_{H}$. If $a \in C_{H}$ then we have $H J_{a} H \subseteq H \subseteq C_{H}$. Furthermore, since $H$ is $\mathscr{S}$ unitary, $H a b H \subseteq H$ if and only if $H a H, H b H \subseteq H$, for $a, b \in S^{1}$. Hence $C_{H}$ is a unitary semigroup, $\bar{C}_{H}$ is an ideal, and $\rho_{H}$ is a nonuniversal semilattice congruence.

(iii) implies (ii). Since a $\mathscr{J}^{*}$-class is $\mathscr{S}$-unitary, it is an ideal. But ideals of $S$ intersect nontrivially.

The next theorem is an immediate consequence of results in [5], [6] or [9].

THEOREM 2.2. For any semigroup $S$ the following are equivalent:

(i) $S$ is an $\mathscr{S}_{1}$-semigroup.

(ii) Each ideal of $S$ is an $\mathscr{S}_{1}$-semigroup.

(iii) $S$ is an ideal extension of an $\mathscr{S}_{1}$-semigroup I by an $\mathscr{S}_{1}$-semigroup $T$.

Note that $T$ has zero divisors.

COROllary 2.3. Let $S$ be a regular semigroup with a principal series. $S$ is a $\mathscr{S}_{1}$-semigroup if and only if each 0 -simple principal factor of $S$ has a zero divisor.

-Proof. By Theorem 2.2, the condition is clearly necessary. 
Conversely let $S_{0} \subset S_{1} \subset \cdots \subset S_{n}=S$ be a principal series. Since $S_{0}$ is simple it is an $\mathscr{S}_{1}$-semigroup. Continuing by induction, assume $S_{i-1}$ is an $\mathscr{S}_{1}$-semigroup and $S_{\imath} / S_{\imath-1}$ has zero divisors for some $i, 1 \leqq i \leqq$ n. $S_{i} / S_{i-1}$ is 0 -simple so is an $\mathscr{S}_{1}$-semigroup. Hence, by Theorem 2.2 (iii), $S_{\imath}$ is an $\mathscr{S}_{1}$-semigroup.

Let $B(G, I)$ denote the Brandt semigroup that is a Rees matrix semigroup over the group with zero $G^{0}$ and with the identity $I \times I$ sandwich matrix. We call $G$ the basic group of $B(G, I) . \quad B(G, I)$ has zero divisors if $|I|>1$ and is isomorphic to $G^{0}$ if $|I|=1$. Since an inverse semigroup is completely [0]-simple if and only if it is a group [Brandt semigroup], we have from Corollary 2.3:

COROLlARY 2.4. Let $S$ be a completely semisimple inverse semigroup with principal series $S_{0} \subset S_{1} \subset \cdots \subset S_{n}=S . \quad S$ is an $\mathscr{S}_{1}$-semigroup if and only if (i) $S_{0}$ is a group, and (ii) $S_{i} / S_{i-1} \cong B\left(G_{i}, I_{i}\right)$ with $\left|I_{i}\right|>1$ for $1 \leqq i \leqq n$.

We conclude this section with a similar result for inverse $\mathscr{I}_{2^{-}}$ semigroups.

THEOREM 2.5. Let $S$ be a completely semisimple inverse semigroup with principal series $S_{0} \subset S_{1} \subset \cdots \subset S_{n}=S . \quad S$ is an $\mathscr{S}_{2}$-semigroup if and only if (i) $S_{0}$ is a group and (ii) $S_{i} / S_{t-1} \cong B\left(G_{i}, I_{i}\right)$ for $1 \leqq i \leqq n$ where $\left|I_{r}\right|=1$ for exactly one $r, 1 \leqq r \leqq n$.

Proof. We first observe that if $\left|I_{r}\right|=1$ and $J_{r}=S_{r} \backslash S_{r-1}, 1 \leqq r \leqq n$, then $J_{r}$ is an $\mathscr{S}$-unitary subgroup of $S$ that is a $\mathscr{J}$-class but not an ideal. As in the proof of Theorem 2.1 there is a semilattice congruence $\rho_{J_{r}}$ with classes $C_{J_{r}}, \bar{C}_{J_{r}}$ defined as in (1).

Assume that $S$ is an $\mathscr{S}_{2}$-semigroup then $S_{0}$ is a group and by Corollary 2.4 there exists an $r, 1 \leqq r \leqq n$, so that $\left|I_{r}\right|=1$. Suppose also that $\left|I_{t}\right|=1,1 \leqq t \leqq n$. Then $\rho_{J_{r}}=\mathscr{J}^{*}=\rho_{J_{t}}$. Hence $C_{J_{r}}=C_{J_{t}}$ and since $J_{r}, J_{t}$ are $\mathscr{J}$-classes, $r=t$.

Conversely assume (i) and (ii). As in the proof of Theorem 2.1, $C_{J_{r}}$ is a unitary subsemigroup and $\bar{C}_{J_{r}}$ is an ideal of $S$. Then the $\mathscr{J}$-classes of $C_{J_{r}}$ and the $\mathscr{J}$-classes of $\bar{C}_{J_{r}}$ are $\mathscr{J}$-classes of $S$. Since $S_{0} \subseteq \bar{C}_{J_{r}}$ and $J_{r} \subseteq C_{J_{r}}$ are the only $\mathscr{J}$-classes that are groups then $\bar{C}_{J_{r}}, C_{J_{r}}$ are $\mathscr{S}_{1^{-}}$ semigroups. Hence $\rho_{J_{r}}=\mathscr{J}^{*}$.

3. Characterization. In this section completely semisimple inverse $\Delta$-semigroups with principal series are characterized.

The following Lemma is an immediate consequence of results of Preston [7]. Parts (i) and (ii) are also corollaries of Tamura [10]. 
Lemma 3.1. Let $S=B(G, I)$ be a Brandt semigroup.

(i) $S$ is $\Delta$-semigroup if and only if $G$ is a $\Delta$-group.

(ii) Each congruence of $S$ is idempotent separating or universal.

(iii) $S$ is primitive.

We need some further results.

Lemma 3.2. Let $S$ be an inverse semigroup with ideal I. Any congruence $\rho^{\prime}$ on I extends to a congruence $\rho$ on $S$ so that

$$
a \rho= \begin{cases}a \rho^{\prime} & \text { if } a \in I \\ \{a\} & \text { if } a \in S \backslash I .\end{cases}
$$

In particular, any ideal of an inverse $\Delta$-semigroup is an inverse $\Delta$ semigroup.

Proof. Let $A$ and $B$ be congruence classes of $\rho^{\prime}$. Suppose xay $\epsilon$ $B$ for some $x, y \in S^{1}, \quad a \in A$. Since $x a a^{-1} a a^{-1} a y \in B$ and $x a a^{-1}, a^{-1} a y \in I$ then $x a a^{-1} A a^{-1} a y \subseteq B$. If $c \in A$ then $a a^{-1} c a^{-1} a \in A$ and $x c c^{-1} a a^{-1} c a^{-1} a c^{-1} c y=x a a^{-1} c a^{-1} a y \in B$ so $x c c^{-1} A c^{-1} c y \subseteq B$. In particular $x c y \in B$. Hence $x A y \subseteq B$. Since $I$ is an ideal the result follows.

If $S$ is an inverse semigroup with semilattice $E$, let $C(E)$ denote the centralizer of $E$ in $S$.

LEMMA 3.3. Let $S$ be a completely semisimple inverse semigroup with principal series $\{0\} \subset S_{1} \subset S$ and with semilattice $E$. Then on $S$;

(i) Each non idempotent separating congruence has $S_{1}$ or $S$ as a congruence class if and only if for any $e, f \in E$ so that $e \in S \backslash S_{1}, f \in S_{1} \backslash 0$ there exists $a \in S$ so that $a^{-1} e a=f$ and so that $f a=0$ if $e>f$.

(ii) Each idempotent separating congruence is the identity equivalence on $S \backslash S_{1}$ if and only if $C(E) \cap\left(S \backslash S_{1}\right) \subseteq E$.

Proof. (i) Suppose the non idempotent separating congruences have $S_{1}$ or $S$ as congruence classes. If $a \in S_{1} \mid 0$ then $S_{1}=J(a)$. If $b \notin J(a)$ then considering the Rees congruence modulo $J(b)$ we see that $J(a) \subset J(b)=S$. Hence the principal ideals of $S$ are chain ordered. Let $\tau$ be the least congruence so that for some $e \neq f$ in $E \backslash 0$, $(e, f) \in \tau$. Assume that $e \in S \backslash S_{1}$ and $f \in S_{1}$. Then $\tau$ is universal. Since $0 \in f \tau$ then by Teissier [12] there exists $x_{1}, y_{1}, \cdots, x_{n}$, $y_{n} \in S^{1}$ so that

$$
f=x_{1} i_{1} y_{1}, \quad x_{1} j_{1} y_{1}=x_{2} i_{2} y_{2}, \cdots, \quad x_{n} j_{n} y_{n}=0
$$


where $i_{p}, j_{p} \in\{e, f\}, \quad p=1, \cdots, n . \quad$ But $\left(x_{p} i_{p} y_{p}\right)^{-1}\left(x_{p} i_{p} y_{p}\right)=z_{p}^{-1} i_{p} z_{p}$ where $z_{p}=x_{p}^{-1} x_{p} y_{p}$. So

$$
f=z_{1}^{-1} i_{1} z_{1}, \quad z_{1}^{-1} j_{1} z_{1}=z_{2}^{-1} i_{2} z_{2}, \cdots, \quad z_{n}^{-1} j_{n} z_{n}=0 .
$$

Deleting repetitious terms we may assume that $z_{p}^{-1} i_{p} z_{p} \neq z_{p}^{-1} j_{p} z_{p}$, and that $z_{p}^{-1} i_{p} z_{p}>z_{p}^{-1} j_{p} z_{p}$ (otherwise replace $z_{q}$ by $z_{q} z_{p}^{-1} i_{p} z_{p}$ for $p \leqq q \leqq n$ ). If $e>f$, then $z_{p}^{-1} e z_{p} \geqq z_{p}^{-1} f z_{p}$, so $i_{p}=e, j_{p}=f$. Furthermore, by Lemma 3.1 (iii) we have $f=z_{1}^{-1} e z_{1}>z_{1}^{-1} f z_{1}=0$. Hence $\left(f z_{1}^{\prime}\right)^{-1}\left(f z_{1}\right)=0$ so $f z_{1}=0$.

Conversely, suppose $e \neq f$ in $E$. If $e \in S \backslash S_{1}$, and $f \in S_{1} \backslash 0$, then $a^{-1} e a=f$ for some $a \in S$, so $J(f) \subset J(e)=S$. Let $\tau$ be the least congruence with $(e, f) \in \tau$. 'By Lemma 3.1 (ii), if $e, f \in S_{1}$, then $e \tau \supseteq$ $S_{1}$. If $e, f \in S \backslash S_{1}$, then, by Lemma 3.1 (iii), ef $\in S_{1}$ and $e f \in e \tau$. If $e \in S \backslash S_{1}$ and $f \in S_{1}$, then $0 \in e \tau$ since either $e>f$ and $0=a^{-1} f a \leqq$ $a^{-1} e a=f$ for some $a \in S$, or $e f=0$ by Lemma 3.1 (iii). Then $e \tau=$ $J(e)=S$.

(ii) By [1] the greatest idempotent separating congruence on $S$ has group kernel normal system $\left\{H_{e} \cap C(E) ; e \in E\right\}$ where $H_{e}$ is the $\mathscr{H}$-class of $e$.

LEMMA 3.4. Let $S$ be a completely semisimple inverse $\mathscr{S}_{1}$-semigroup with principal series $\{0\} \subset S_{1} \subset S . \quad S$ is a $\Delta$-semigroup if and only if

(i) the Brandt semigroups $S / S_{1}$ and $S_{1}$ have $\Delta$-basic groups,

(ii) each non idempotent separating congruence of $S$ has $S_{1}$ or $S$ as a congruence class, and

(iii) each idempotent separating congruence of $S$ is the identity equivalence on $S \backslash S_{1}$.

Proof. Let $S$ be a $\Delta$-semigroup. By Lemmas 3.1 (i) and 3.2, (i) is satisfied. Comparing congruences with the Rees congruence modulo $S_{1}$ we see that (iii) holds and that any non universal congruence has its classes in $S_{1}$ or $S \backslash S_{1}$. Hence, applying Lemma 3.1 (ii) to $S_{1}$, we see that (ii) holds.

Conversely, by (i), (iii) and Lemma 3.1 (i) applied to $S_{1}$, the idempotent separating congruences are chain ordered. By (ii) the other non universal congruences have $S_{1}$ as a class and are then chain ordered since, by (i), $S / S_{1}$ is a $\Delta$-semigroup. Hence, by (iii), $S$ is a $\Delta$-semigroup.

LEMMA 3.5. Let $S$ be a completely semisimple inverse $\mathscr{S}_{2}$-semigroup with principal series $\{0\} \subset S_{1} \subset S . \quad S$ is a $\Delta$-semigroup if and only if $S_{1}$ is an $\mathscr{S}_{1}$ - $\Delta$-semigroup, $S \backslash S_{1}$ is a $\Delta$-group and $S$ satisfies conditions (ii) and (iii) of Lemma 3.4.

Proof. By Theorem 2.5 just one of $S_{1} \backslash 0$ or $S \backslash S_{1}$ is a 
group. Assume $S$ is a $\Delta$-semigroup. The $\mathscr{J}$-classes of $S$ are chain ordered [11]. If $S_{1} \mid 0$ is a group then, as in the proof of Theorem $2.5, \mathscr{J}^{*}$ has classes $\{0\}, S \backslash 0$. But then $\mathscr{J}^{*}$ is not comparable with the Rees congruence modulo $S_{1}$. Hence $S \backslash S_{1}$ is a group while $S_{1} \backslash 0$ is not. The remainder of the proof is as for Lemma 3.4.

The following theorem is the main result. Together with the results $2.4,2.5,3.1(\mathrm{i}), 3.3,3.4$ and 3.5 , it provides a characterization of completely semisimple inverse $\Delta$-semigroups with principal series in terms of $\Delta$-groups and idempotent properties.

THEOREM 3.6. Let $S$ be a completely semisimple inverse semigroup with principal series $S_{0} \subset S_{1} \subset \cdots \subset S_{n}=S . \quad S$ is a $\Delta$-semigroup if and only if

(i) $S_{0}$ is a $\Delta$-group; $S_{0}=\{0\}$ if $n>0$,

(ii) $S_{1}$ is a Brandt semigroup with $\Delta$-basic group if $n>0$,

(iii) $S_{t} / S_{t-2}$ is an $\mathscr{S}_{1}-\Delta$-semigroup for $i=2, \cdots, n-1$,

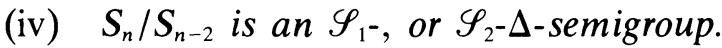

Proof. Say $S$ is a $\Delta$-semigroup. $S$ and $S_{0}$ have the same maximal group homomorphic image and if $n>0$ the only such group is trivial [11]. Hence, by Lemmas 3.2, 3.1(i), (i), 3.4 and 3.5, we see that (i), $\cdots$ (iv) are satisfied.

Conversely we prove that for any congruence $\rho$ on $S$ and some $i$, $0 \leqq i \leqq n$, then $a \rho=S_{i}$ for $a \in S_{i}, e \rho \cap E=\{e\}$ for $e \in\left(S_{i+1} \mid S_{i}\right) \cap E$ where $E$ is the semilattice of $S$, and $a \rho=\{a\}$ for $a \in S \backslash S_{i+1}$. Then $S$ will be a $\Delta$-semigroup. The result holds for $n=0$ or 1 , by Lemma 3.1 (ii). Continue by induction, assuming the result for $n=t$. Since $S_{t+1} / S_{t-1}$ is a $\Delta$-semigroup, then the congruences of $S_{t+1}$ that have their classes in $S_{t}$ or $S_{t+1} \backslash S_{t}$ are of the required form by Lemmas 3.4, 3.5. Suppose $\rho$ is a congruence on $S_{t+1}$ with $(a, b) \in \rho, a \in S_{t+1} \mid S_{t}$, $b \in S_{t}$. Then the congruence on the $\Delta$-semigroup $S_{t+1} / S_{t-1}$ induced by $\rho$ is universal by Lemmas 3.4, 3.5. Hence there exists $h \in S_{t} \backslash S_{t-1}, k \in S_{t-1}$ so that $(h, k) \in \rho$. But then, by the induction assumption, $S_{t} \subseteq h \rho$. Since $S_{t+1} / S_{t-1}$ is a $\Delta$-semigroup, then, by Lemmas 3.4, 3.5, a $\rho=S_{t+1}$.

4. Further study of finite case. We now investigate circumstances under which the extensions of Theorem 3.6 are possible for finite inverse semigroups. Some further information is required.

EXAMPLE 1. Let $H_{X}$ be the subgroup of the symmetric group $P_{X}$ whose elements displace a finite number of elements of the set $X$. The alternating group $A_{X}$ is a simple normal subgroup of $H_{X}$ with index 2 (see [3]) for $|X| \neq 4$. Hence $H_{X}$ is a $\Delta$-group. In particular if $|X|$ is finite then $P_{X}$ is a $\Delta$-group. 
EXAMPLE 2. The symmetric inverse semigroup $\mathscr{I}_{X},|X|$ finite, is a $\Delta$-semigroup. To see this, let $D(\alpha), R(\alpha)$ denote the domain and range of $\alpha \in \mathscr{I}_{X}$ respectively. Any ideal of $\mathscr{I}_{X}$ is of the form $I_{n}=\left\{\alpha \in \mathscr{I}_{X}\right.$; $|D(\alpha)| \leqq n\}$. Since $\mathscr{I}_{X}$ is finite it has a principal series and is completely semisimple. If $\alpha$ is an idempotent its $\mathscr{H}$-class is $\left\{\beta \in \mathscr{I}_{X} ; D(\beta)=\right.$ $R(\beta)=D(\alpha)\}$ (see [4]), which is the symmetric group on $D(\alpha)$. So for some $\alpha$ a non group principal factor has $\Delta$-basic group isomorphic to $P_{D(\alpha)}$. If $\alpha, \gamma$ are idempotents so that $|D(\alpha)|>|D(\gamma)| \geqq 1$ then there is a $\beta \in \mathscr{I}_{X}$ so that $\beta^{-1} \alpha \beta=\gamma$ and $\left|D\left(\beta^{-1} \gamma \beta\right)\right|<|D(\gamma)|$. If $\alpha$ is not an idempotent there is an idempotent $\beta$ so that $|D(\alpha)|-|D(\beta)| \leqq 1$ and $\beta \alpha \neq \alpha \beta . \quad \mathscr{I}_{X}$ can now be seen to satisfy the requirements of Lemmas 3.3, 3.4 and Theorem 3.6.

Let $Z_{n}$ denote the set $\{1,2, \cdots, n\}$. If $X=Z_{n}$ write $P_{n}=P_{X}$ and $\mathscr{I}_{n}=\mathscr{I}_{X}$.

Suppose $S$ is a finite $\Delta$-semigroup with $\{0\} \subset S_{1} \subset S, S_{1} \cong B\left(G, Z_{n}\right)$ and $S / S_{1} \cong B\left(H, Z_{r}\right)$. Let $\left(G \times Z_{n} \times Z_{n}\right) \cup\{0\}$ denote the set of elements of $S_{1}$, with the binary operation $(x, i, j)(y, h, k)=(x y, i, k)$ if $j=h$, and 0 if $j \neq h$.

Denote the semigroup of right translations of $S_{1}$ by $P\left(S_{1}\right)$ and for $a \in S$ define $\rho^{a} \in P\left(S_{1}\right)$ by $b \rho^{a}=b a$ for all $b \in S_{1}$. Since inverse semigroups are left reductive there is a unique homomorphism $\theta: S \rightarrow P\left(S_{1}\right)$ so that the restriction of $\theta$ to $S_{1}$ is the regular representation of $S_{1}$ (by [6; III.1.12]). $\theta$ is given by $a \theta=\rho^{a}, a \in S$, and $\left(S_{1}\right) \theta \cong S_{1}$. Since $S$ is a $\Delta$-semigroup then, by Lemma 3.4 or $3.5, \theta$ is injective. Call $\theta$ the extension homomorphism of $S$.

Let 1 denote the identity of $G$. For $u \in S, i \in Z_{n}$ define $D(u)=$ $\left\{j \in Z_{n} ;(1, i, j) u \theta \neq 0\right\} . \quad$ By $\left[6 ; V .3 .6\right.$ and V.5.4] there exists $\phi_{u} \in \mathscr{I}_{n}$ with domain $D\left(\phi_{u}\right)=D(u)$ and a map $a_{u}: D(u) \rightarrow G$ so that

$$
(x, i, j) u \theta= \begin{cases}\left(x\left(j a_{u}\right), i, j \phi_{u}\right) & \text { if } j \in D(u), \\ 0 & \text { if } j \notin D(u) .\end{cases}
$$

Furthermore the map given by $u \theta \rightarrow\left(a_{u}, \phi_{u}\right)$ defines an isomorphism between $(S) \theta$ and the semigroup $\left\{\left(a_{u}, \phi_{u}\right) ; u \in S\right\}$ with the binary operation $\left(a_{u}, \phi_{u}\right)\left(a_{v}, \phi_{v}\right)=\left(a_{u} \cdot a_{v}, \phi_{u} \phi_{v}\right)$ where $j\left(a_{u} \cdot a_{v}\right)=\left(j a_{u}\right)\left(j \phi_{u} a_{v}\right)$. Since $\theta$ is an isomorphism then $\left(a_{u v}, \phi_{u v}\right)=\left(a_{u} \cdot a_{v}, \phi_{u} \phi_{v}\right)$. Note that with the operations - and composition of maps, the sets $\left\{a_{u} ; u \in S\right\}$ and $\left\{\phi_{u} ; u \in S\right\}$ respectively are homomorphic images of $S$. For convenience we will identify $u \theta$ and $\left(a_{u}, \phi_{u}\right)$ for each $u \in S$.

Clearly $v \in S_{1}$ if and only if $\left|D\left(\phi_{v}\right)\right| \leqq 1$. Since $\phi_{u}$ is a bijection for $u \in S$ then $\left\{v \in S ;\left|D\left(\phi_{v}\right)\right| \leqq\left|D\left(\phi_{u}\right)\right|\right\}$ is an ideal of $S$. Hence for $u, v \in S \backslash S_{1}, \quad\left|D\left(\phi_{u}\right)\right|=\left|D\left(\phi_{v}\right)\right| ; \quad$ call this number the rank of $S / S_{1}$. Clearly $e$ is an idempotent of $S \backslash 0$ if and only if $\left(D\left(\phi_{e}\right)\right) a_{e}=\{1\}$ 
and $\phi_{e}$ is an identity map. A product of distinct idempotents $e, f \in S$ is in $S_{1}$ so $\left|D\left(\phi_{e}\right) \cap D\left(\phi_{f}\right)\right| \leqq 1$. Hence if $S \backslash S_{1}$ is not a group then the rank of $S / S_{1}$ is bounded above by $[(n+1) / 2]$.

Definition 4.1. For integers $m$ and $n, 1<m \leqq n$, let ${ }^{n} \Gamma_{m}$ denote the largest number so that $\left\{Y_{i} ;\left|Y_{i}\right|=m, i=1, \cdots,{ }^{n} \Gamma_{m}\right\}$ is a family of subsets of $Z_{n}$ with $\left|Y_{i} \cap Y_{j}\right| \leqq 1$ for $i \neq j$. For an integer $r, 1<r \leqq{ }^{n} \Gamma_{m}$, let $\mathscr{A}=\left\{X_{i} ;\left|X_{i}\right|=m, i \in Z_{r}\right\}$ be a family of subsets of $Z_{n}$ with $\left|X_{i} \cap X_{j}\right| \leqq 1$ for $i \neq j$. Let

$$
\mathscr{A}^{*}=\left\{\alpha \in \mathscr{J}_{n} ; \alpha=0 \quad \text { or } \quad D(\alpha), R(\alpha) \in \mathscr{A}\right\}
$$

with a binary operation $*$ so that

$$
\alpha * \beta= \begin{cases}\alpha \beta & \text { if } R(\alpha)=D(\beta) \\ 0 & \text { if } R(\alpha) \neq D(\beta) .\end{cases}
$$

LEMMA 4.2. Let $S$ be a $\Delta$-semigroup with principal series $\{0\} \subset S_{1} \subset S$ so that $S_{1} \cong B\left(G, Z_{n}\right)$ and $S / S_{1} \cong B\left(H, Z_{r}\right)$ has rank $m$. Then $r=1$

(i) either $1<m \leqq[(n+1) / 2]$ and $1<r \leqq{ }^{n} \Gamma_{m}$ or $1<m \leqq n$ and

(ii) $H$ is embeddable in the symmetric group $P_{m}$.

Proof. Part (i) follows from the preceding commets and definition. Let $Q=\left(S \backslash S_{1}\right) \cup\{0\}$ and define a binary operation $*$ so that $u * v=u v$ if $u v \in S \backslash S_{1}$, and 0 otherwise. Then $Q \cong B\left(H, Z_{r}\right)$. Let $\mathscr{A}=\left\{D\left(\phi_{u}\right) ; u \in S \backslash S_{1}\right\}$. The map $\delta: Q \rightarrow \mathscr{A}^{*}$ given by $u \delta=\phi_{u}$ is a homomorphism. If $u \neq v$ in $S \backslash S_{1}$ and $\phi_{u}=\phi_{v}$ then $\left|D\left(\phi_{u v^{-1}}\right)\right|>1$ so $u v^{-1}$ is a non idempotent element of $S \backslash S_{1}$. But then for any idempotent $e \in S$, it can be readily shown that $\left(e u v^{-1}\right) \theta=\left(u v^{-1} e\right) \theta$ so $e u v^{-1}=$ $u v^{-1} e$. This contradicts Lemmas 3.3 and 3.4 or 3.5. Hence $\delta$ is injective. If $e \neq 0$ is an idempotent of $Q$ then it can be easily shown that the $\mathscr{H}$-class of $e$ in $Q$ is $H_{e}=\left\{u \in Q ; D\left(\phi_{u}\right)=R\left(\phi_{u}\right)=D\left(\phi_{e}\right)\right\}$. Then $\left(H_{e}\right) \delta \cong H_{e} \cong H$. Part (ii) follows since the elements of $\left(H_{e}\right) \delta$ are permutations of $D\left(\phi_{e}\right)$.

Let $\mathscr{B}^{*}=\left\{a_{u} ; u \in S \backslash S_{1}\right\} \cup\{0\}$ with a binary operation $*$ so that $a_{u} * a_{v}=a_{u} \cdot a_{v}$ if $u v \in S \backslash S_{1}$, and 0 otherwise. Since $\delta$ is injective, if $\phi_{u}=\phi_{v}$ for $u, v \in S \backslash S_{1}$ then $u=v$ so $a_{u}=a_{v}$. Hence there is a homomorphism $\lambda:(Q) \delta \rightarrow \mathscr{B}^{*}$ given by $\phi_{u} \lambda=a_{u}$ if $u \in S \backslash S_{1}$ and $0 \lambda=$ 0 . The set $\vec{H}=\{(u \delta \lambda, u \delta) ; u \in Q\}$ with the binary operation so that $(u \delta \lambda, u \delta)(v \delta \lambda, v \lambda)=((u * v) \delta \lambda,(u * v) \delta)$ is then a semigroup isomorphic to $Q$. 
Definition 4.3. A structure data set is a set $\{n, r, m, G, \bar{H}\}$ defined as follows:

(i) $n, r$ and $m$ are integers so that either $1<m \leqq[(n+1) / 2]$ and $1<r \leqq{ }^{n} \Gamma_{m}$, or $1<m \leqq n$ and $r=1$.

(ii) $G$ is a $\Delta$-group.

(iii) Let $\mathscr{A}=\left\{X_{i} ;\left|X_{i}\right|=m, i \in Z_{r}\right\}$ be a family of subsets of $Z_{n}$ so that $\left|X_{\imath} \cap X_{j}\right| \leqq 1$ if $i \neq j$. Let $H$ be a $\Delta$-subgroup of the symmetric group $P_{m}$. Let $K$ be a subsemigroup of $\mathscr{A}^{*}$ so that $K \cong B\left(H, Z_{r}\right)$. Let $\lambda: K \rightarrow \mathscr{B}^{*}$ be a surjective homomorphism so that for $\phi \in K$, $\phi \lambda: D(\phi) \rightarrow G$ is a map and so that for $j \in D(\phi * \psi)$ then $j(\phi * \psi) \lambda=$ $(j(\phi \lambda))(j \phi(\psi \lambda))$. Define $\bar{H}=\{(\phi \lambda, \phi) ; \phi \in K\}$ with a binary operation so that $(\phi \lambda, \phi)(\psi \lambda, \psi)=((\phi * \psi) \lambda, \phi * \psi)$. Write $\phi \lambda * \psi \lambda=(\phi * \psi) \lambda$.

Notice that in the terminology of [6], $\bar{H}$ satisfies this definition if and only if $\bar{H}$ is a subsemigroup of the right wreath product of $G$ and $K$ so that the map $\bar{H} \rightarrow K$ given by $(\phi \lambda, \phi) \rightarrow \phi$ is an isomorphism.

We have seen that any finite inverse $\Delta$-semigroup $S$ with principal series $\{0\} \subset S_{1} \subset S$ determines a structure data set $\{n, r, m, G, \bar{H}\}$. Call this a structure data set of $S$. We say that structure data sets $\{n, r, m, G, \bar{H}\}$ and $\left\{n^{\prime}, r^{\prime}, m^{\prime}, G^{\prime}, \bar{H}^{\prime}\right\}$ are equivalent if and only if $n=n^{\prime}$, $r=r^{\prime}, m=m^{\prime}$ and there exists an isomorphism $\alpha: G^{\circ} \rightarrow\left(G^{\prime}\right)^{\circ}$ and a bijection $\beta: Z_{n} \rightarrow Z_{n}$ so that the map $\gamma: \bar{H} \rightarrow \bar{H}^{\prime}$ given by $(a, \phi) \gamma=$ $\left(\beta^{-1} a \alpha, \beta^{-1} \phi \beta\right)$ is a bijection.

LEMMA 4.4. Let $S$ and $T$ be finite inverse $\Delta$-semigroups with principal series $\{0\} \subset S_{1} \subset S$ and $\{0\} \subset T_{1} \subset T$ respectively. Then $S \cong T$ if and only if the structure data sets of $S$ and $T$ are all equivalent.

Proof. Lable the elements of $S_{1}$ and $T_{1}$ so that $S_{1}=$ $\left(G \times Z_{n} \times Z_{n}\right) \cup\{0\}$ and $T_{1}=\left(G^{\prime} \times Z_{n^{\prime}} \times Z_{n^{\prime}}\right) \cup\{0\}$, with binary operations as defined after Example 2. Then structure data sets $\{n, r, m, G, \bar{H}\}$ and $\left\{n^{\prime}, r^{\prime}, m^{\prime}, G^{\prime}, \bar{H}^{\prime}\right\}$ of $S$ and $T$ respectively can be uniquely determined by the method described above. Depending on the labelling of the elements of $S_{1}$, each structure data set of $S$ can be so determined. Let $\theta_{S}$ and $\theta_{T}$ be the extension homomorphisms of $S$ and $T$ respectively and let $\eta: S \rightarrow T$ be an isomorphism. Then $n=n^{\prime}, r=r^{\prime}$ and the restriction of $\eta$ to $S_{1}$ determines an isomorphism $\alpha: G^{\circ} \rightarrow\left(G^{\prime}\right)^{\circ}$ and a bijection $\beta: Z_{n} \rightarrow Z_{n}$ so that $(x, i, j) \eta=(x \alpha, i \beta, j \beta) \in T_{1}$. The map $u \theta_{s}, u \in S$, is given by $v\left(u \theta_{S}\right)=v u$ for all $v \in S_{1}$. Let $u \eta \theta_{T}=\left(b_{u \eta}, \psi_{u \eta}\right)$ and $v=(x, i, j)$ then

$$
\left(\left(x\left(j a_{u}\right)\right) \alpha, i \beta, j \phi_{u} \beta\right)=\left(v\left(u \theta_{s}\right)\right) \eta=(v \eta)\left(u \eta \theta_{T}\right)=\left((x \alpha) j \beta b_{u \eta}, i \beta, j \beta \psi_{u \eta}\right) .
$$

So $\left(x\left(j a_{u}\right)\right) \alpha=\left(x\left(j \beta b_{u \eta}\right) \alpha^{-1}\right) \alpha$. Since $D(u) \beta=D(u \eta)$ then $a_{u}=$ 
$\beta b_{u \eta} \alpha^{-1}$. Likewise $\phi_{u} \beta=\beta \psi_{u \eta}$. Thus $m=m^{\prime}$ and since $\bar{H}=$ $\left\{\left(a_{u}, \phi_{u}\right) ; u \in S \backslash S_{1}\right\} \cup\{0\}$ then the structure data sets are equivalent.

Conversely, given that $\{n, r, m, G, \bar{H}\}$ and $\left\{n^{\prime}, r^{\prime}, m^{\prime}, G^{\prime}, \bar{H}^{\prime}\right\}$ are equivalent structure data sets, let $\alpha: G^{\circ} \rightarrow\left(G^{\prime}\right)^{\circ}$ be an isomorphism, $\beta: Z_{n} \rightarrow Z_{n}$ be a bijection and $\gamma: \bar{H} \rightarrow \bar{H}^{\prime}$ be the bijection so that $(a, \phi) \gamma=\left(\beta^{-1} a \alpha, \beta^{-1} \phi \beta\right)$. Define $\eta_{1}: S_{1} \rightarrow T_{1}$ by $(x, i, j) \eta_{1}=(x \alpha, i \beta, j \beta)$. Then $\eta_{1}$ is an isomorphism. As in the first part of the proof we get for $v \in S_{1}$ that $v \eta_{1} \theta_{T}=\left(\beta^{-1} a_{v} \alpha, \beta^{-1} \phi_{v} \beta\right)$. Hence there is a bijection $\gamma^{\prime}:(S) \theta_{S} \rightarrow(T) \theta_{T}$ given by

$$
\left(a_{u}, \phi_{u}\right) \gamma^{\prime}=\left(\beta^{-1} a_{u} \alpha, \beta^{-1} \phi_{u} \beta\right) .
$$

Define $\eta: S \rightarrow T$ by $u \eta \theta_{T}=u \theta_{S} \gamma^{\prime}$. Then

$$
\begin{aligned}
(x, i, j) \eta_{1}\left(u \eta \theta_{T}\right) & =\left(x \alpha\left(j \beta \beta^{-1} a_{u} \alpha\right), i \beta, j \beta \beta^{-1} \phi_{u} \beta\right)=\left(\left(x\left(j a_{u}\right)\right) \alpha, i \beta, j \phi_{u} \beta\right) \\
& =(x, i, j) u \theta_{s} \eta_{1} .
\end{aligned}
$$

So $u \eta \theta_{T}=\eta_{1}^{-1}\left(u \theta_{S}\right) \eta_{1}$ and clearly $\eta$ is an isomorphism.

THEOREM 4.5. Each finite inverse $\Delta$-semigroup $S$ with principal series $\{0\} \subset S_{1} \subset S$ has a structure data set $\{n, r, m, G, \bar{H}\}$. A semigroup is isomorphic to $S$ if and only if its structure data sets are equivalent to $\{n, r, m, G, \bar{H}\}$. Conversely, each structure data set $\{n, r, m, G, \bar{H}\}$ is a structure data set of some finite inverse $\Delta$-semigroup $T$ with principal series $\{0\} \subset T_{1} \subset T$.

Proof. The first two statements have been proved. Suppose $\{n, r, m, G, \bar{H}\}$ is a structure data set. Let $T_{1}=\left(G \times Z_{n} \times Z_{n}\right) \cup\{0\}$ with binary operation as defined after Example 2. Then $T_{1} \cong B\left(G, Z_{n}\right)$. Let $T=T_{1} \cup \bar{H} \backslash(0,0)$. For $(a, \phi),(b, \psi) \in \bar{H} \backslash(0,0)$ and $(x, i, j),(y, h, k) \in T_{1}$ define a binary operation on $T$ so that:

$$
\begin{aligned}
& (a, \phi)(b, \psi)= \begin{cases}(a * b, \phi \psi) \in \bar{H} & \text { if } \quad D(\phi \psi)=D(\phi) \\
(l a(l \phi a), l, l \phi \psi) \in T_{1} & \text { if } \quad D(\phi \psi)=\{l\} \\
0 & \text { if } D(\phi \psi)=\square,\end{cases} \\
& (x, i, j)(a, \phi)= \begin{cases}(x(j a), i, j \phi) \in T_{1} & \text { if } j \in D(\phi) \\
0 & \text { if } \quad j \notin D(\phi),\end{cases} \\
& (a, \phi)(x, i, j)= \begin{cases}\left(i \phi^{-1} a x, i \phi^{-1}, j\right) \in T_{1} & \text { if } i \in R(\phi) \\
0 & \text { if } \quad i \notin R(\phi),\end{cases}
\end{aligned}
$$




$$
(x, i, j)(y, h, k)=\left\{\begin{array}{lll}
(x y, i, k) \in T_{1} & \text { if } \quad j=h \\
0 & \text { if } j \neq h .
\end{array}\right.
$$

Since $\bar{H} \cong B\left(H, Z_{r}\right)$ it can be routinely checked that $T$ is an inverse semigroup. It can also be checked, using Lemmas 3.3 and 3.4 or 3.5, that $S$ is a $\Delta$-semigroup. Since $(x, i, j)(a, \phi)=(x(j a), i, j \phi)$ for $(x, i, j) \in T_{1},(a, \phi) \in T / T_{1}$ and $j \in D(\phi)$ we see that $\{n, r, m, G, \bar{H}\}$ is a structure data set of $T$.

Let $S$ be a finite inverse $\Delta$-semigroup with principal series $S_{0} \subset S_{1} \subset$ $\cdots \subset S_{q}=S$ where $q>1$. We can uniquely determine, up to equivalence, the structure data sets of the semigroups $S_{i} / S_{i-2}$ for $i=$ $2, \cdots, q$. Conversely, let $\left\{\left\{n_{i}, r_{i}, m_{i}, G_{i}, \bar{H}_{i}\right\} ; i=2, \cdots, q\right\}$ be a family of structure data sets so that $n_{j}=r_{j-1}$ and $G_{j} \cong H_{j-1}$ for $j=3, \cdots, q$, where $H_{j-1}$ is the basic group of $\bar{H}_{j-1}$. Then, by Theorem 3.6 and the proof of Theorem 4.5 , we can construct a finite inverse $\Delta$-semigroup $T$ with principal series $T_{0} \subset T_{1} \subset \cdots \subset T_{q}=T$ so that $\left\{n_{i}, r_{i}, m_{i}, G_{i}, \bar{H}_{i}\right\}$ is a structure data set of $T_{t} / T_{t-2}$. Any finite inverse $\Delta$-semigroup that is not a group or a Brandt semigroup can be so constructed.

The authors are grateful for the referee's kind suggestions on this paper.

\section{REFERENCES}

1. A. H. Clifford, and G. B. Preston, The algebraic theory of semigroups, Math. Surveys No. 7, Amer. Math. Soc., Providence, Vol. I (1961), Vol. II (1967).

2. J. M. Howie, and G. Lallement, Certain fundamental congruences on a regular semigroup, Proc. Glasgow Math. Assoc., 7 (1966), 145-156.

3. M. Hall Jr., The Theory of Groups, Macmillan, New York (1959).

4. W. D. Munn, Fundamental inverse semigroups, Quart. J. Math. Oxford (2), 21 (1970), 157-170.

5. M. Petrich, The maximal semilattice decomposition of a sẻmigroup, Math. Zeitschr., 85 (1964), 68-82.

6. _ Introduction to semigroups, Merrill, Columbus (1973).

7. G. B. Preston, Congruences on Brandt semigroups, Math. Ann., 139 (1959), 91-94.

8. B. M. Schein, Commutative semigroups whose congruences form a chain, Polonaise des Sciences, 9 (1969), 523-527.

9. T. Tamura, The theory of construction of finite semigroups I, Osaka Math. J., 8 (1956), 243-261.

10. - Decomposition of a completely simple semigroup, Osaka Math. J., 12 (1960), 269-275.

11. Commutative semigroups whose lattice of congruences form a chain, Bull. Soc. Math. France, 97 (1969), 369-380.

12. M. Teissier, Sur les équivalences regulières dans les demi-groupes, C. R. Acad. Sci., Paris, 232 (1951), 1987-1989.

Received November 7, 1975 and in revised form September 13, 1976.

The University of Tasmania, Hobart, Australia

AND

The University of CALIFornia,

DAVIS, CA 95616 



\section{PACIFIC JOURNAL OF MATHEMATICS}

\section{EDITORS}

RichaRd ARENS (Managing Editor)

University of California

Los Angeles, CA 90024

\section{R. A. Beaumont}

University of Washington

Seattle, WA 98105

C. C. Moore

University of California

Berkeley, CA 94720
J. DugunduI

Department of Mathematics

University of Southern California

Los Angeles, CA 90007

R. Finn AND J. Milgram

Stanford University

Stanford, CA 94305

\section{ASSOCIATE EDITORS}
E. F. BECKENBACH
B. H. NeumanN
F. WOLF
K. YoshidA

\section{SUPPORTING INSTITUTIONS}

UNIVERSITY OF BRITISH COLUMBIA

CALIFORNIA INSTITUTE OF TECHNOLOGY

UNIVERSITY OF CALIFORNIA

MONTANA STATE UNIVERSITY

UNIVERSITY OF NEVADA

NEW MEXICO STATE UNIVERSITY

OREGON STATE UNIVERSITY

UNIVERSITY OF OREGON

OSAKA UNIVERSITY

\author{
UNIVERSITY OF SOUTHERN CALIFORNIA \\ STANFORD UNIVERSITY \\ UNIVERSITY OF HAWAII \\ UNIVERSITY OF TOKYO \\ UNIVERSITY OF UTAH \\ WASHINGTON STATE UNIVERSITY \\ UNIVERSITY OF WASHINGTON \\ AMERICAN MATHEMATICAL SOCIETY
}

The Supporting Institutions listed above contribute to the cost of publication of this Journal, but they are not owners or publishers and have no responsibility for its contents or policies.

Mathematical papers intended for publication in the Pacific Journal of Mathematics should be in typed form or offset-reproduced (not dittoed), double spaced with large margins. Underline Greek letters in red, German in green, and script in blue. The first paragraph or two must be capable of being used separately as a synopsis of the entire paper. Items of the bibliography should not be cited there unless absolutely necessary, in which case they must be identified by author and Journal, rather than by item number. Manuscripts, in duplicate, may be sent to any one of the four editors. Please classify according to the scheme of Math. Reviews, Index to Vol. 39. All other communications should be addressed to the managing editor, or Elaine Barth, University of California, Los Angeles, California, 90024.

100 reprints are provided free for each article, only if page charges have been substantially paid. Additional copies may be obtained at cost in multiples of 50 .

The Pacific Journal of Mathematics is issued monthly as of January 1966. Regular subscription rate: $\$ 72.00$ a year (6 Vols., 12 issues). Special rate: $\$ 36.00$ a year to individual members of supporting institutions.

Subscriptions, orders for back numbers, and changes of address should be sent to Pacific Journal of Mathematics, 103 Highland Boulevard, Berkeley, California, 94708.

PUBLISHED BY PACIFIC JOURNAL OF MATHEMATICS, A NON-PROFIT CORPORATION

Printed at Jerusalem Academic Press, POB 2390, Jerusalem, Israel. 


\section{Pacific Journal of Mathematics}

Vol. 68, No. 2

April, 1977

William Allen Adkins, Aldo Andreotti and John Vincent Leahy, An analogue of Oka's theorem for weakly normal complex spaces ........

Ann K. Boyle, M. G. Deshpande and Edmund H. Feller, On nonsingularly

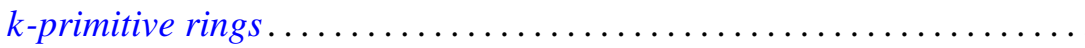

Rolando Basim Chuaqui, Measures invariant under a group of

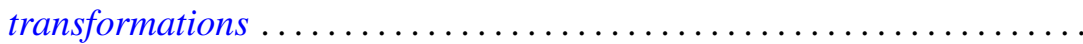

Wendell Dan Curtis and Forrest Miller, Gauge groups and classification of bundles with simple structural group .......................

Garret J. Etgen and Willie Taylor, The essential uniqueness of bounded nonoscillatory solutions of certain even order differential equations

Paul Ezust, On a representation theory for ideal systems

Richard Carl Gilbert, The deficiency index of a third order operator ........

John Norman Ginsburg, $S$-spaces in countably compact spaces using Ostaszewski's method.

Basil Gordon and S. P. Mohanty, On a theorem of Delaunay and some

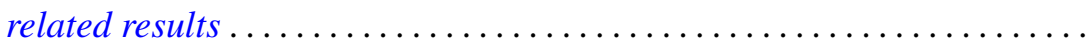

Douglas Lloyd Grant, Topological groups which satisfy an open mapping

theorem.

Charles Lemuel Hagopian, A characterization of solenoids

Kyong Taik Hahn, On completeness of the Bergman metric and its

subordinate metrics. II .

G. Hochschild and David Wheeler Wigner, Abstractly split group extensions.

Gary S. Itzkowitz, Inner invariant subspaces ...............

Jiang Luh and Mohan S. Putcha, A commutativity theorem for

non-associative algebras over a principal ideal domain.

Donald J. Newman and A. R. Reddy, Addendum to: "Rational approximation of $e^{-x}$ on the positive real axis".....

Akio Osada, On the distribution of a-points of a strongly annular function ....

Jeffrey Lynn Spielman, A characterization of the Gaussian distribution in a Hilbert space. .

Robert Moffatt Stephenson Jr., Symmetrizable-closed spaces ...

Peter George Trotter and Takayuki Tamura, Completely semisimple inverse $\Delta$-semigroups admitting principal series . . . . . . . .

Charles Irvin Vinsonhaler and William Jennings Wickless, Torsion free abelian groups quasi-projective over their endomorphism rings...

Frank Arvey Wattenberg, Topologies on the set of closed subsets ... 